\title{
The management of ureteric stones in the Accident and Emergency department
}

\author{
Kirsty Dawson, Lindsay Dawson \\ Croydon University Hospital
}

\begin{abstract}
Ureteric stones is a common cause for attendance to the A\&E department. Active or conservative management is delivered dependent on the relevant history, clinical condition, and investigation results. The majority of patients can be treated conservatively and do not require admission. However, some cases of ureteric stones present as a urological emergency if, for example, there is an infected obstructed system. An initial audit over a two month period of $A \& E$ admissions with radiologically proven ureteric stones demonstrated there was no easily accessible, specific criteria used to guide which patients were admitted and which patients were discharged from A\&E. Therefore, an admission criteria and discharge proforma was developed and implemented in the A\&E department to ensure patients were appropriately discharged if they could be managed conservatively and appropriately admitted if they potentially required further observation and intervention. A repeat audit using the same methods and criteria was carried out and demonstrated fewer unnecessary admissions and fewer inappropriate discharges.
\end{abstract}

\section{Problem}

Ureteric stones make up a significant proportion of acute attendances to hospital.(1) However, many patients do not require admission to hospital. There does not always seem to be a logic behind which patients with ureteric stones are admitted and which are discharged. It was felt that by setting out criteria to be used in the A\&E department, differentiating patients requiring admission for further observation and management, and patients who could be safely discharged, it would avoid unnecessary hospital admissions and reduce inappropriate discharges.

\section{Background}

Ureteric stones are a common problem. Stones are responsible for more than 12,000 hospital admissions each year.(2)

The majority of ureteric stones less than $8 \mathrm{~mm}$ will pass spontaneously without intervention.(3) The British Association of Urological Surgeons (BAUS) state only three indications for intervention in the management of ureteric stones: uncontrolled pain, infected obstructed kidney, single kidney. The BAUS guidelines state that patients should be admitted with ureteric stones if there are signs of obstruction and also state that a urgent urological opinion should be sought in patients with, uncontrolled pain, signs of sepsis or a single kidney.(4) However, if an infected obstructed kidney is not urgently treated, rapid nephron destruction can occur.

If active management is not indicated, the European Association of Urology (EUA) state that patients with newly diagnosed ureteric stones less than $10 \mathrm{~mm}$ should be offered conservative medical expulsive management, consisting of analgesia and an alphablocker.(5) However, this treatment does not necessarily need to be carried out in hospital.(6)

Between $5-12 \%$ of the population will have a urinary tract stone during their lifetime, and recurrence rates approach $50 \%$.(7) Therefore, inappropriate management of ureteric stones could not only have a potentially huge impact on cost implications for hospital trusts but can also lead to major complications for patients.

\section{Baseline Measurement}

A preliminary audit was undertaken over a two month period (September-October 2012) at Croydon University Hospital, a district general hospital in South London. Patients aged over 18 years, diagnosed with an image proven ureteric stone were identified. Using the hospital computer records the presenting complaint, observations, blood results, and imaging were reviewed. 28 patients with a mean age of 42.8 (range 19-73), fulfilled the criteria. Of these 28 patients, 15 were admitted and 13 were discharged from A\&E. Using the BAUS guidelines, the outcomes for this group of patients were deemed appropriate or inappropriate. The BAUS guidelines state that patients should be admitted with ureteric stones if there are signs of obstruction, uncontrolled pain, signs of sepsis, or a single kidney.

Of the 15 patients admitted, 2 admissions (13.3\%) were deemed inappropriate (i.e. these patients could have been safely discharged with conservative management). Of the 13 patients discharged, 4 discharges (30.8\%) were deemed inappropriate (i.e. these patients would have been more appropriate for admission for further observation). These 4 inappropriate discharges included 3 patients with acutely deranged renal function and 1 patient with signs of sepsis.

Overall, this preliminary audit demonstrated that 6 patients (21.4\%) had inappropriate outcomes. 
See supplementary file: ds2469.doc - "Preliminary Audit Results"

\section{Design}

It was felt that there were two issues to address in order to improve the appropriateness of patients' outcomes. Firstly, A\&E staff needed to be made more aware of and have easy access to guidelines identifing criteria for admission and discharge for patients with ureteric stones. Secondly, the guidelines needed to be more objective than the BAUS guidelines. For example, the guidelines need to be more specific than, 'admission is necessary if signs of sepsis or signs of obstruction.'

Therefore, a proforma was designed with a traffic light system of criteria. If all the 'green' criteria are met, a patient can be discharged, and if any of the 'red' criteria are met a patient needs referral to the surgical admission team. Based upon the BAUS guidelines, specific criteria for admission and discharge was decided and agreed upon in conjunction with the urology consultants and registrars.

The 'green' criteria for discharge included normal urea and electrolytes and full blood counts, stone diameter less than $5 \mathrm{~mm}$, adequate pain relief and the patient being a pyrexial. The 'red' criteria for admission included a stone diameter greater than $5 \mathrm{~mm}$, inadequate pain relief, creatinine greater than 125, a single kidney, temperature greater than 37.5 degrees, a white cell count greater than 14.0, or clinical signs of sepsis (hypotension, tachycardia).

A traffic light system was used to make the guidelines easy to interpret and with specific criteria, easy to follow. The proforma was uploaded on to the trust's intranet site, making it readily available on any hospital computer. It was felt this was more appropriate and sustainable than having temporary posters in the department.

\section{Strategy}

PDSA cycle 1: The results of the initial audit were discussed at the weekly urology multi-disciplinary team (MDT) meeting. The criteria for admission and discharge were discussed with the urology consultants and registrars. The design for the proforma was also shown to the MDT and finalised with their suggestions. For example, advice regarding pain management was added to the proforma and advice to give to patients upon discharge from A\&E.

PDSA cycle 2: The results of the initial audit were discussed at the monthly general surgery and urology clinical governance meeting and the proforma was introduced to the surgical junior doctors. The surgical SHOs on-call also cover urology admissions so it was important that they were aware of the new proforma and accepted the guidelines. Whilst the criteria for admission and discharge were accepted, the junior doctors felt that if a patient with a ureteric stone presented with an infected obstructed system, a urological emergency, the proforma should outline how to contact the urology registrar or consultant on-call directly. This was agreed and added to the proforma.
PDSA cycle 3: The completed proforma was then introduced to the A\&E consultants and juniors and they were shown how to access the proforma from the intranet. An issue brought up during this meeting with $A \& E$ staff was that junior staff often rotate every 4 or 6 months, therefore, incoming staff would not be aware of the proforma. To solve this problem, during the induction for new staff to the $A \& E$ department, the proforma and other guidelines are now introduced to staff and how to access them.

Subsequently the proforma was rolled-out and a further audit was carried out after 8 months.

\section{Results}

8 months following the introduction and rolling-out of the proforma a re-audit was carried out using the same methods as the initial audit. Over a two month period (August-September 2013) at Croydon University Hospital, patients aged over 18 years, diagnosed with an image proven ureteric stone were identified. Using the hospital computer records the presenting complaint, observations, blood results, and imaging were reviewed.

21 patients with a mean age of 43.0 (range 21-73), fulfilled the criteria. Of these 21 patients, 7 were admitted and 14 were discharged from A\&E. Using the criteria from the proforma based upon the BAUS guidelines, the outcomes for this group of patients were deemed appropriate or inappropriate.

Of the 7 patients admitted, all admissions (100\%) were deemed appropriate. Of the 14 patients discharged, 3 discharges $(21.4 \%)$ were deemed inappropriate. These 3 inappropriate discharges included 2 patients that had a white cell count greater than 14.0 and 1 patient who had a image proven ureteric stone greater than $5 \mathrm{~mm}$. According to the proforma, these 3 patients required admission.

Overall, the re-audit demonstrated that 3 patients (14.3\%) had inappropriate outcomes. These results demonstrated that there were no inappropriate admissions, therefore, reducing unnecessary bed-days. The results also demonstrated a reduction in inappropriate discharges, therefore, an improvement in patient safety.

See supplementary file: ds2468.doc - "Re-audit Results"

\section{Lessons and Limitations}

The number of cases that were reviewed during the preliminary and repeat audit was small. If a bigger sample size had been used the results would be more robust.

Although there was improvement demonstrated in the results of the re-audit, there were still some patients who were discharged inappropriately. The potential reasons for this were analysed and a problem was identified.

A number of patients who attend $A \& E$ with a provisional diagnosis of ureteric stones are often discharged and advised to re-attend the 


\section{BMJ Quality Improvement Reports}

department the following day for imaging to confirm or exclude a ureteric stone. This occurs more out-of-hours when imaging availability is limited. Patients are only discharged to have imaging the following day if they meet the other 'green' criteria on the proforma, i.e. if a patient has normal renal function, no signs of infection, and controlled pain.

However, there seems to be a problem in reviewing the images of these patients. The imaging needs to be reviewed to ensure there is not an obstructed system or there is not a large stone, which may not pass spontaneously. To ensure patients are not missed following their imaging, written advice is now given to patients advising they return to the A\&E department to be reviewed by a doctor along with the results of the imaging.

The proforma that was designed gives specific values as part of the admission and discharge criteria. For example, a white cell count greater than 14.0 requires admission or a creatinine greater than 125. However, clinical judgement needs to be applied where a patient presents with a white cell count of 13.9 for example.

With the introduction of stricter admission criteria, there have been cases of re-attendances to A\&E. This has mainly been due to pain control and none of the re-attendances went on to be admitted. However, it has highlighted that when discharging patients from A\&E, they need to be properly counselled and their expectations managed regarding the course of their acute illness.

\section{Conclusion}

This project aimed to improve the appropriateness of admissions and discharges from A\&E for patients with ureteric stones. By designing and introducing a proforma, which set out criteria to guide which patients required admission and which patients could be safely discharged, an improvement has been demonstrated.

The intervention is sustainable and cheap and has reduced the number of unnecessary bed-days, and improved patient safety by reducing the number of inappropriate discharges.

\section{References}

1. Raj P, Killian Mellon J. (2008). Renal stone disease. The Foundation Years, 4(5): 199-203.

2. BAUS website. http://www.baus.org.uk/patients/symptoms/calculi. Accessed 22nd October 2013.

3. Coll D, Varanello M, Smith R. (2002). Relationship of spontaneous passage of ureteral calculi to stone size and location as revealed by unenhanced helical CT. ALR Am J Roentgenol, 178(1): 101-103.

4. Parys B, McClinton S, Watson G, Anson K, Burgess N, Dickinson A, et al. (2009). BAUS Section of Endourology Guidelines for Actue Management of First Presentation of Renal/Ureteric Lithiasis, Journal of Clinical Urology, 2(3): 134-136.

5. Turk C, Knoll T, Petrik A, Sarica K, Skolarikos A, Straub M, et al. (2013). Guidelines on Urolithiasis. European Association of Urology.

6. Morris S, Hampson S, Gordon E, Shearer R, Woodhouse C. (1995). Should all patients with ureteric colic be admitted? Ann R Coll Surg Engl, 77(6): 450-452.

7. Sierakowski R, Finlayson B, Landes R R. (1978). The frequency of urolithiasis in hospital discharge diagnoses in the United States. Invest Urol, 15(6): 438-441.

\section{Declaration of interests}

Nothing to declare

\section{Acknowledgements}

Mr N Arsanious, Miss T Nitkunan, Dr K Channing 